\title{
„wer wen besiegt, das intressiert auch mich“ \\ Brechts Laotse: weiser Held und Stratege des gewaltlosen Widerstands
}

\section{Dieter Martin}

Bertolt Brecht hat sich - angeregt durch Richard Wilhelms epochemachende Übersetzung von Laotses Tao Te King (1911), Alfred Döblins ,chinesischen Roman“ Die drei Sprünge des Wang-lun (1916) und Klabunds unter dem barockisierenden Titel Mensch, werde wesentlich! erschienene Nachdichtungen der Sprüche des Laotse (1920) - erstmals um 1920 eingehend mit dem Taoismus befasst. Doch blieb dies bei Brecht nicht eine bloß kurzzeitige Begeisterung für die damalige Modephilosophie aus dem Fernen Osten. Vielmehr lassen sich, das haben Heinrich Detering und Karl-Josef Kuschel in ihren 2008 bzw. 2018 erschienenen Monographien eindrücklich nachgezeichnet, ${ }^{1}$ Kernelemente der taoistischen Lehre, wie vor allem das antagonistische Bild von dem das Harte überwindenden weichen Wasser als langjährige, über alle biographischen Brüche sowie ideologischen Wenden hinwegreichenden Konstanten in Brechts Denken und Dichten erkennen. Seine Auseinandersetzung mit dem Taoismus kulminiert fraglos die in der 1939 in den Svendborger Gedichten veröffentlichten Legende von der Entstehung des Buches Taoteking auf dem Weg des Laotse in die Emigration:

1

Als er siebzig war und war gebrechlich

Drängte es den Lehrer doch nach Ruh

Denn die Güte war im Lande wieder einmal schwächlich

Und die Bosheit nahm an Kräften wieder einmal zu.

5 Und er gürtete den Schuh.

2

Und er packte ein, was er so brauchte:

Wenig. Doch es wurde dies und das.

So die Pfeife, die er immer abends rauchte

1 Heinrich Detering: Bertolt Brecht und Laotse, Göttingen 2008; Karl-Josef Kuschel: Im Fluss der Dinge. Hermann Hesse und Bertolt Brecht im Dialog mit Buddha, Laotse und Zen, Ostfildern 2018, bes. S. 537-646. - Zu Brechts Rezeption Chinas und speziell der taoistischen Philosophie vgl. zuvor Antony Tatlow: The Mask of Evil. Brecht's Response to the Poetry, Theatre and Thought of China and Japan. A Comparative and Critical Evaluation, Bern u. a. 1977; Yun-Yeop Song: Bertolt Brecht und die chinesische Philosophie, Bonn 1978; Han-Soon Yim: Bertolt Brecht und sein Verhältnis zur chinesischen Philosophie, Bonn 1984; Weijian Liu: Die daoistische Philosophie im Werk von Hesse, Döblin und Brecht, Bochum 1991; Christoph Gellner: Weisheit, Kunst und Lebenskunst. Fernöstliche Religion und Philosophie bei Hermann Hesse und Bertolt Brecht, Mainz 1997. 
Und das Büchlein, das er immer las.

10 Weißbrot nach dem Augenmaß.

3

Freute sich des Tals noch einmal und vergaß es

Als er ins Gebirg den Weg einschlug.

Und sein Ochse freute sich des frischen Grases

Kauend, während er den Alten trug.

15 Denn dem ging es schnell genug.

4

Doch am vierten Tag im Felsgesteine

Hat ein Zöllner ihm den Weg verwehrt:

„Kostbarkeiten zu verzollen?“ - „Keine.“

Und der Knabe, der den Ochsen führte, sprach: „Er hat gelehrt.“

20 Und so war auch das erklärt.

5

Doch der Mann in einer heitren Regung

Fragte noch: „Hat er was rausgekriegt?“

Sprach der Knabe: „Daß das weiche Wasser in Bewegung

Mit der Zeit den mächtigen Stein besiegt.

25 Du verstehst, das Harte unterliegt.“

6

Daß er nicht das letzte Tageslicht verlöre

Trieb der Knabe nun den Ochsen an.

Und die drei verschwanden schon um eine schwarze Föhre

Da kam plötzlich Fahrt in unsern Mann

30 Und er schrie: „He du! Halt an!

7

Was ist das mit diesem Wasser, Alter?““

Hielt der Alte: „Intressiert es dich?“

Sprach der Mann: „Ich bin nur Zollverwalter

Doch wer wen besiegt, das intressiert auch mich.

35 Wenn du's weißt, dann sprich!

8

Schreib mir's auf! Diktier es diesem Kinde!

So was nimmt man doch nicht mit sich fort.

Da gibt's doch Papier bei uns und Tinte

und ein Nachtmahl gibt es auch: ich wohne dort.

40 Nun, ist das ein Wort?"“

9

Über seine Schulter sah der Alte

Auf den Mann: Flickjoppe. Keine Schuh.

Und die Stirne eine einzige Falte.

Ach, kein Sieger trat da auf ihn zu.

45 Und er murmelte: „Auch du?“ 
10

Eine höfliche Bitte abzuschlagen

War der Alte, wie es schien, zu alt.

Denn er sagte laut: „Die etwas fragen

Die verdienen Antwort.“ Sprach der Knabe: „Es wird auch schon kalt.“

50 „Gut, ein kleiner Aufenthalt.“

11

Und von seinem Ochsen stieg der Weise

Sieben Tage schrieben sie zu zweit.

Und der Zöllner brachte Essen (und er fluchte nur noch leise

Mit den Schmugglern in der ganzen Zeit.)

55 Und dann war's soweit.

12

Und dem Zöllner händigte der Knabe

Eines Morgens einundachtzig Sprüche ein

Und mit Dank für eine kleine Reisegabe

Bogen sie um jene Föhre ins Gestein.

60 Sagt jetzt: kann man höflicher sein?

13

Aber rühmen wir nicht nur den Weisen

Dessen Name auf dem Buche prangt!

Denn man muß dem Weisen seine Weisheit erst entreißen.

Darum sei der Zöllner auch bedankt:

65 Er hat sie ihm abverlangt. ${ }^{2}$

Das in trochäischen Drei- bis Achthebern verfasste Gedicht, das in dreizehn, schon in der Erstausgabe durchnummerierte Strophen aus Kreuzreimen mit angehängtem fünften Vers $(a b a b b)$ gegliedert ist, zählt zu Brechts bekanntesten lyrischen Werken. Entsprechend oft ist das balladeske Erzählgedicht, das im Wechsel aus präteritalem Erzählerbericht mit szenischen Dialogen die im Titel genannte Entstehungslegende vorstellt und abschließend in eine präsentische Höreransprache mündet, schon gedeutet worden - am intensivsten sicher in den Monographien von Detering und Kuschel, die Brechts textliche und bildliche Vorlagen bestimmen und ausführlich die geistesgeschichtlichen Kontexte sowie die Textgenese rekonstruieren. ${ }^{3}$

2 Bertolt Brecht: Gedichte 2. Sammlungen 1938-1956, bearb. von Jan Knopf (B. B.: Werke. Große kommentierte Berliner und Frankfurter Ausgabe 12), Berlin u. a. 1988, S. 32-34. Zitate aus dem Gedicht werden durch Angabe der Verszahl im laufenden Text nachgewiesen (Abweichungen zur Zeilenzählung in der zitierten Ausgabe ergeben sich dadurch, dass dort sowohl der Gedichttitel als auch die Strophennummern mitgezählt sind).

3 Detering: Brecht (Anm. 1); Kuschel: Fluss (Anm. 1), hier S. 569-618. Die wesentliche Bildquelle für Brechts Gedicht, eine in Richard Wilhelms Übersetzung wiedergegebene chinesische Zeichnung, findet sich bei Detering: Brecht (Anm. 1), S. 8-9, und Kuschel: Fluss (Anm. 1), Abb. 6 (Tafelteil nach S. 360). Beide zitieren auch ausführlich aus Wilhelms Vorwort, das als zentrale Textquelle für Brechts Kenntnis der Entstehungslegende gelten darf; vgl. Detering: Brecht (Anm. 1), S. 14, und Kuschel: Fluss (Anm. 1), S. 551-552. - Aus der Forschungsliteratur (weitere Titel bei Kuschel: Fluss [Anm. 1], S. 685) seien ferner ge- 
Erstaunlich wenig Anregung bietet die Forschung indessen, wenn man das Gedicht unter dem für den vorliegenden Sammelband maßgeblichen Aspekt der Heroisierung einer fremdkulturellen Geistesgröße zu lesen unternimmt. Die banalste Erklärung dafür wäre sicher die, dass das Gedicht nun einmal keine Heldengeschichte erzähle, sondern eher das Gegenteil davon: Ein weiser Lehrer, der den misslichen Umständen in seinem „Lande“ entflieht, weil es ihn „nach Ruh“ drängt (2-3), und der seine Heimat scheinbar leichthin vergessen kann (11), schreibt seine bislang nur mündlich mitgeteilte Lehre einzig deshalb nieder, weil ihn ein Zöllner aus einer ,heitren Regung" heraus darum bittet (21) und ihm dafür einen bequemen „Aufenthalt“ (50) mit einem „Nachtmahl“ anbietet (39). So paraphrasiert, klingt das kaum nach einer heroischen Tat. ${ }^{4}$ Und doch: Zieht man das Publikationsumfeld von Brechts Legende, die vor allem im dänischen Exil verfassten Svendborger Gedichte hinzu, dann gerät man unabweisbar in einen heroischen Diskurs. Das gilt besonders für die das Laotse-Gedicht enthaltende Abteilung der Chroniken, den dritten Abschnitt der Sammlung. Während der erste Teil, die Deutsche Kriegsfibel, in epigrammatischer Nüchternheit die gegensätzliche Realität des Krieges für die Oberen und die Niedrigen markiert und der zweite Teil in Gestus und Form populäre Lieder und Balladen über die gegenwärtigen und älteren „finsteren Zeiten“ enthält, während die vierte Abteilung aufrührerische Appelle aufbietet, die fünfte Deutsche Satiren über Hitlers Regime versammelt und die sechste einen Epilog aus Reflexionen bildet, darunter die berühmten Verse An die Nachgeborenen, sind die Chroniken des dritten Abschnitts wenigstens zu einem guten Teil als propagandistische Lehrgedichte über einen proletarischkommunistischen Heroismus und als Reflexionen über die Geschichtsmacht des Einzelnen respektive der Masse zu lesen. ${ }^{5}$ Schon das Eingangsgedicht der Chroniken negiert mit seinen Fragen eines lesenden Arbeiters die Heroengeschichtsschrei-

nannt: Peter Horst Neumann: Der Weise und der Elefant. Zwei Brecht-Studien, München 1970, hier S. 65-75; Antony Tatlow: Towards an Understanding of Chinese Influence in Brecht. An Interpretation of ,Auf einen chinesischen Theewurzellöwen' and ,Legende von der Entstehung des Buches Taoteking', in: Deutsche Vierteljahrsschrift für Literaturwissenschaft und Geistesgeschichte 44, 1970, S. 363-387, hier S. 374-387; Walter Hinck: Der Weise und der Wißbegierige, in: Jan Knopf (Hg.): Gedichte von Bertolt Brecht, Stuttgart 1995, S. 133-146; Jan Knopf: Gelegentlich: Poesie. Ein Essay über die Lyrik Bertolt Brechts, Frankfurt am Main 1996, hier S. 160-166; Antony Tatlow: Legende von der Entstehung des Buches Taoteking, in: Jan Knopf (Hg.): Brecht-Handbuch, Bd. 2: Gedichte, Stuttgart/ Weimar 2001, S. 299-302; Ulrich Kittstein: Das lyrische Werk Bertolt Brechts, Stuttgart/ Weimar 2012, hier S. 167-173.

4 Vgl. Hinck: Der Weise und der Wißbegierige (Anm. 3), S. 134: „Brecht macht aus dem Emigrationsanlaß und aus Laotses Entschluß, das Land zu verlassen, keine heroische Situation, aus dem Gang ins Exil keine abenteuerliche Flucht.“ Ebd., S. 135: „Und doch ist diese ,Chronik' keine Wiederherstellung der alten Heldenballade mit ausgetauschtem Personal.“

5 Brecht: Gedichte 2 (Anm. 2), S. 7-92, zitiert S. 16 (Motto zu Abt. II); zur Entstehung ebd., S. 349-358. Zum Aufbau und zur formalen wie gehaltlichen Vielfalt der Svendborger Gedichte vgl. bes. Knopf: Poesie (Anm. 3), S. 141-198. Zum ,heroischen' Umfeld der Legende siehe auch Detering: Brecht (Anm. 1), S. 67-68. 
bung, die personal verkürzt den ,jungen Alexander [...] Indien“ erobern und „Cäsar [...] die Gallier" besiegen lässt. ${ }^{6}$ Gegen diesen traditionellen Heldenkult berichten die meist in reimlosen freien Rhythmen verfassten Chroniken von proletarischer Kameradschaft, von erfolgreicher Sabotage, vom aktiven Widerstand gegen die Macht des Kapitals und davon, wie unter Lenin und Stalin ein Ausgleich zwischen individuellem und kollektivem Heroismus gelinge. So feiert die Moskauer Arbeiterschaft die Inbesitznahme der großen Metro als Sieg ihrer Tatkraft über „[a]lle Schwierigkeiten“.7 So können Die Teppichweber von Kujan-Bulak [...] Lenin gerade dadurch ebren, dass sie das für eine Lenin-Büste gesammelte Geld doch lieber in Petroleum investieren, mit dem sie die umliegenden Sümpfe von Stechmücken reinigen. ${ }^{8}$ Und so wird Der große Oktober, Brechts Beitrag Zum zwanzigsten Jahrestag der Oktoberrevolution, zum Sinnbild einer Selbstermächtigung der „Arbeiterklasse“ verklärt: der „unendliche Zug der Sieger“ werfe allen „Unterdrückern“ die „Drohung“ der kommenden Weltrevolution entgegen. ${ }^{9}$

Wie aber passt die Laotse-Legende in den Kontext dieser massiven Propaganda, dieser eindeutigen, vor dem zeitgeschichtlichen Hintergrund von Faschismus und Kriegstreiberei begreiflichen Parteinahme Brechts, dieser Appelle, die der Arbeiterklasse aufzeigen wollen, dass heroische Tatkraft und kollektives Handeln sie zum Sieg führe?

Ansätze zur möglichen Erklärungen ergeben sich, wenn man darauf achtet, dass und wie Brecht das Porträt des legendären chinesischen Philosophen einerseits als Bild kultureller Alterität entwirft, in das er andererseits aber deutliche Züge der Aneignung, ja der persönlichen Identifikation einzeichnet. Einerseits: Als kulturell Anderer und Fremdartiger erscheint Laotse schon insofern, als die Legende über seinen Weg [...] in die Emigration die sonst in den Chroniken genutzten Mittel historischer Beglaubigung ausspart. Wo andere Gedichte konkrete Orte und Daten nennen, ${ }^{10}$ um die prosanahen Berichte faktual zu beglaubigen, da spielt die Laotse-Legende in geographischer und historischer Ferne, in einer fast noch archaischen Vorzeit. Zwar gibt es rudimentäre soziale Organisationsformen mit Grenzen und Zöllnern, jede Konkretisierung der „im Lande“ herrschenden „Bosheit“ (3-4) fehlt aber. So fügt sich das in gereimten Versen und regelmäßigen Strophen poetisierte Exempel nicht in die sonst in den Chroniken vorherrschenden Antithesen von Kommunismus und Kapital. Andererseits aber passt Brecht

Brecht: Gedichte 2 (Anm. 2), S. 29, zit. V. 15 und 17.

Ebd., S. 43-45, zit. V. 8.

Ebd., S. 37-39.

Ebd., S. 45-46, zit. V. 2, 26 und 43-44.

10 Z. B. ebd., S. 39 (Die unbesiegliche Inschrift, Eingangsverse): „Zur Zeit des Weltkriegs / In einer Zelle des italienischen Gefängnisses San Carlo [...]"; S. 40 (Koblen für Mike, Eingangsverse): „Ich habe gehört, daß in Ohio / Zu Beginn des Jahrhunderts [...]"); S. 41 (Abbau des Schiffes Oskawa durch die Mannschaft, Eingangsverse): „Zu Beginn des Jahres 1922 / Nahm ich Heuer auf dem Sechstausendtonnendampfer OSKAWA“. 
das Bild Laotses an kulturell vertraute Vorstellungen an, die mit der Realität im alten China nichts zu tun haben, und er malt es geradezu als identifikatorisches Selbstporträt aus: So wenig „Weißbrot“, „Büchlein“ und „Pfeife“ (8-10) einem alten chinesischen Weisen zugehören, so deutlich lassen diese anachronistischen Attribute den passionierten Raucher und Leser Brecht durchschimmern. ${ }^{11}$

Funktional mag man diese palimpsestartige Überblendung des Fremden mit dem Eigenen als Versuch Brechts werten, sein individuelles, äußerlich betrachtet, nur wenig heroisches Verhalten zu reflektieren und zu legitimieren. Denn immerhin unterwarf sich der exilierte Brecht nicht dem Terror seiner stalinistischen Gesinnungsgenossen, sondern lebte, wie das Motto der Svendborger Gedichte sagt, „Geflüchtet unter das dänische Strohdach", und er kämpfte nicht etwa im Untergrund oder im spanischen Bürgerkrieg, sondern „[v]erfolg[te]“ aus der Ferne lediglich den „Kampf“" der „Freunde“. 12

Zieht man diese Linie einer biographisch funktionalisierten Einfärbung weiter aus, dann relativiert Brechts Laotse-Legende den in anderen Chroniken propagierten Kollektivheroismus durch ein identifikatorisches Bekenntnis zum individuellen Eskapismus. Dass Brecht die Figur des historisch fernen Anti-Helden Laotse dazu nutzt, eigene öffentlich bekundete Positionen zu korrigieren und deren Geltung einzuschränken, legt auch die im Titel hervorgehobene Charakterisierung von Laotses Weg als einen in die Emigration nahe - reflektiert Brecht doch in der sechsten Abteilung der Svendborger Gedichte Über die Bezeichnung Emigranten, um diese als Euphemismus abzulehnen: „Immer fand ich den Namen falsch, den man uns gab: Emigranten. / Das heißt doch Auswandrer. Aber wir / Wanderten doch nicht aus [...]. / Sondern wir flohen. Vertriebene sind wir, Verbannte."13 Während Laotse die Heimat anscheinend umstandslos vergessen kann (11), harren die Exilanten des Reflexionsgedichts „möglichst nahe den Grenzen / Wartend des Tags der Rückkehr". ${ }^{14}$ Auch zeigt das Schlussgedicht der Svendborger Gedichte, die um Verständnis werbende Anrede An die Nachgeborenen, mit seinen Querbezügen zu Laotses Lehre an, dass diese ein Potenzial bereithält, das Brecht wenigstens als Optativ bewahren möchte, selbst wenn sie in den „finsteren Zeiten“, in denen er lebt, als Irrealis erscheinen muss:

Ich wäre gerne auch weise

In alten Büchern steht, was weise ist:

Sich aus dem Streit der Welt halten und die kurze Zeit

Ohne Furcht verbringen

Auch ohne Gewalt auskommen

Böses mit Gutem vergelten

Seine Wünsche nicht erfüllen, sondern vergessen

Gilt für weise.

11 Vgl. Detering: Brecht (Anm. 1), S. 70-71, und Kuschel: Fluss (Anm. 1), S. 584-585.

12 Brecht: Gedichte 2 (Anm. 2), S. 7.

13 Ebd., S. 81; vgl. Detering: Brecht (Anm. 1), S. 69.

14 Brecht: Gedichte 2 (Anm. 2), S. 81. 
Alles das kann ich nicht:

Wirklich, ich lebe in finsteren Zeiten! ${ }^{15}$

Unterstreicht Brecht einerseits die Alterität des exotisch-fernen Laoste zu dem in der Gegenwart nötigen aktiven Kollektivheroismus, so rückt er die Figur andererseits nicht nur identifikatorisch an sich selbst heran, sondern verbindet sie auch sowohl mit abendländischen Traditionen wie mit dem Diskurs aktueller Politik. Eine Anknüpfung an westliches Denken erreichen die zitierten Verse, indem der (hier ganz fraglos mitgemeinte) Laotse nicht beim Namen genannt und gerade dadurch in den größeren Kontext einer Ethik der Gewaltlosigkeit gestellt wird: Wie die Formulierung „Böses mit Gutem vergelten“16 auf die Antithese zu Beginn der Laotse-Legende verweist („die Güte war [...] schwächlich | Und die Bosheit nahm [...] zu" [3-4]), so alludiert sie auch die neutestamentarische Lehre der Feindesliebe. Mit dieser Assimilierung von Taoismus und Christentum im Zeichen der pazifistischen Ethik greift Brecht das Erfolgsrezept der modernen Laotse-Rezeption auf. So hat der lange Jahre als evangelischer Missionar in Tsingtau wirkende Richard Wilhelm, dessen Laotse-Übertragung Brecht als Hauptquelle diente, die chinesischen Weisheiten dadurch unters deutsche Volk gebracht, dass er typische Redewendungen der Lutherbibel adaptierte und Laotses Lehren damit sprachlich den Schein des Vertrauten gab. ${ }^{17}$

Brecht geht aber insofern deutlich weiter, als seine scheinbar historisch ferne $L e$ gende die Kampfrhetorik der Gegenwart aufnimmt. Im Zentrum des Gedichts, exakt in der mittleren siebten Strophe, lässt Brecht den einfachen Zöllner sein „plötzlich[es]“ Feuer (29) für die vom Knaben zitierte Lehre des Laotse so begründen: „wer wen besiegt, das intressiert auch mich“ (34). Mit dieser auffälligen Wendung, die Subjekt und Objekt eines Entscheidungskampfes engstmöglich verknüpft, hat Brecht eine damals berühmte Formel Lenins adaptiert: „Wer wird wen überflügeln? Gelingt es den Kapitalisten, sich früher zu organisieren, dann werden sie die Kommunisten zum Teufel jagen, darüber braucht man überhaupt kein Wort zu verlieren. Man muß diese Dinge nüchtern betrachten: Wer - wen?"18 Darauf gestoßen, dass Brecht die taoistische Maxime der Gewaltlosigkeit provokativ mit Lenins Analyse machtpolitischer Effizienz verbindet, bemerkt man, wie gezielt er die Laotse-Legende mit einer teleologischen Perspektive, mit der Frage nach Effekt und Erfolg unterlegt. Denn vom ,Sieg' ist nicht nur in der zentralen siebten Stro-

15 Ebd., S. 85-87 (An die Nachgeborenen), hier Abt. I, V. 3 und 24-33.

16 Ebd., V. 29. Vgl. Detering: Brecht (Anm. 1), S. 59.

17 Vgl. Detering: Brecht (Anm. 1), S. 22-28, und Kuschel: Fluss (Anm. 1), S. 27-31.

18 Aus Lenins Referat über Die Neue Ökonomische Politik und die Aufgaben der Ausschüsse für politisch-kulturelle Aufklärung, gehalten am 17. Oktober 1921 auf dem 2. Gesamtrussischen Kongress der Ausschüsse für politisch-kulturelle Aufklärung; zitiert nach Brecht: Gedichte 2 (Anm. 2), S. 368. - Detering: Brecht (Anm. 1), S. 79, und Kuschel: Fluss (Anm. 1), S. 593, erwähnen das Lenin-Zitat zwar, marginalisieren es aber in ihren Deutungen. Interpretatorisch angemessen integriert ist die „Anspielung auf Lenin“, mit der Brecht „durch die Lehre seines Laotse eine Botschaft über den Klassenkampf durchscheinen“ lasse, hingegen bei Kittstein: Werk (Anm. 3), S. 170. 
phe die Rede, sondern in symmetrischer Rahmung auch in der fünften und neunten: „Daß das weiche Wasser in Bewegung / Mit der Zeit den mächtigen Stein besiegt“ (23-24), heißt es dort, und hier: „Ach, kein Sieger trat da auf ihn zu.“ (44) Während die fünfte Strophe auf das plötzlich erwachende Interesse des Zöllners vorausdeutet, das sich nicht am Mittel, sondern am Ausgang des Kampfes entzündet, ${ }^{19}$ motiviert Brecht mit der neunten Strophe, warum sich Laotse die Niederschrift abbitten lässt: Da der Zöllner, wie er selbst, „kein Sieger“ ist (44) und gerade deshalb ein berechtigtes Interesse an einer Lehre hat, die einen Weg zum Erfolg aufzeigt, fühlt sich Laotse dem einfachen Mann solidarisch verbunden und verpflichtet, seine Weisheiten schriftlich festzuhalten.

Wie wichtig Brecht die Akzentuierung des Sieges als langfristiges Ziel gewesen ist, ${ }^{20}$ zeigt sich daran, dass er die neunte Strophe erst nachträglich in das abgeschlossene, bereits durchgezählte Typoskript eingefügt und so die symmetrische Perspektive auf den Sieg des „wer“ über den „wen“ (34), des vermeintlich Schwachen über den angeblich Starken betont hat. ${ }^{21}$ Das nachgetragene, im Gedichtverlauf aber vorangestellte Motiv der Solidarität des Intellektuellen mit dem einfachen Mann war Brecht offenbar so wichtig, dass er dessen Spannung zu dem zuvor schon formulierten Handlungsantrieb in der 10. Strophe in Kauf genommen hat: „Eine höfliche Bitte abzuschlagen / War der Alte, wie es schien, zu alt“ (46-47), so wird Laotses Einwilligung dort begründet - ähnlich vage wie schon in einer Prosaversion der Entstehungslegende, die Brecht bereits 1925 unter dem Titel Die höflichen Chinesen vorgelegt hatte. ${ }^{22}$ Die Pointe der Legende lag für Brecht damals darin,

19 Neben Kuschel: Fluss (Anm. 1), S. 591-600, betonen die Rolle des Zöllners auch Hinck: Der Weise und der Wißbegierige (Anm. 3), und Kittstein: Werk (Anm. 3), S. 167-170, der die Legende wesentlich anhand der „ideale[n] Lehrer-Schüler-Konstellation“ interpretiert und als „Brechts wohl bedeutendstes Gedicht über das Lehren und Lernen“ bezeichnet.

Dass Brecht dasjenige, „was bei Laotse das bloße Konstatieren des unaufhaltsamen Naturrhythmus ist“, „gezielt dynamisier[e] und damit geschichtlich-politisch akzentuier[e]“, betont Kuschel: Fluss (Anm. 1), S. 608, anhand eines detaillierten Textvergleichs zwischen Laotses Spruch 78 (in Wilhelms Übersetzung) und der 5. Strophe in Brechts Legende.

21 Ausführlich zur Textgeschichte vgl. Detering: Brecht (Anm. 1), S. 75-81 und S. 91-93 (mit Abbildungen und Transkriptionen der Typoskripte sowie der handschriftlichen Korrekturen Brechts).

22 Bertolt Brecht: Prosa 4: Geschichten, Filmgeschichten, Drehbücher 1913-1939, bearb. von Brigitte Bergheim (B. B.: Werke. Große kommentierte Berliner und Frankfurter Ausgabe 19), Berlin u. a. 1997, S. 200 (Die höflichen Chinesen; zuerst im Berliner Börsen-Courier von 1925): „Wenigen bekannt in unserer Zeit ist es, wie sehr ein der Allgemeinheit geleisteter Dienst der Entschuldigung bedarf. So ehrten die höflichen Chinesen ihren großen Weisen Laotse, mehr als meines Wissens irgendein anderes Volk seinen Lehrer, durch die Erfindung folgender Geschichte. Laotse hatte von Jugend auf die Chinesen in der Kunst zu leben unterrichtet und verließ als Greis das Land, weil die immer stärker werdende Unvernunft der Leute dem Weisen das Leben erschwerte. Vor die Wahl gestellt, die Unvernunft der Leute zu ertragen oder etwas dagegen zu tun, verließ er das Land. Da trat ihm an der Grenze des Landes ein Zollwächter entgegen und bat ihn, seine Lehren für ihn, den Zollwächter aufzuschreiben, und Laotse, aus Furcht, unhöflich zu erscheinen, willfahrte ihm. Er schrieb die Erfahrungen seines Lebens in einem dünnen Buche für den Zollwächter auf und verließ erst, als es geschrieben war, das Land seiner Geburt. Mit dieser Geschichte ent- 
dass Laotse der Bitte des Zöllners entsprochen habe, weil er fürchtete sonst „unhöflich zu erscheinen“, und dass man in dieser (,in unserer Zeit" merkwürdig wirkenden) Motivation das Bedürfnis der „höflichen Chinesen“ erkennen könne, einen „der Allgemeinheit geleistete[n] Dienst“, hier speziell: „das Zustandekommen des Buches Taoteking“ zu „entschuldigen“. ${ }^{23}$ Dass sich aber die topische Höflichkeit der Chinesen gerade mit der Legende von der Entstehung von Laotses Buch exemplifizieren ließ, dürfte daran liegen, dass es einer „Entschuldigung“24 für die schriftliche Fixierung speziell jener Weisheitslehre bedurfte, die so einzigartig das Fluide betont, die daher gerade nicht auf schriftliche Fixierung und erst recht nicht auf Verdauerung ihres individuellen Schöpfers zielt, der sich ja auch in Brechts Gedicht dem Vergessen anheimgeben will.

Brechts Legende von der Entstehung des Buches Taoteking zeigt sich so als ein Text, der von inhärenten Ambivalenzen geprägt ist und zugleich erheblich in Spannung zur agitatorischen Propaganda anderer Chroniken der Svendborger Gedichte steht. Mit ihrer kulturellen Alterität und ihrem antiheroischen Bekenntnis zur Gewaltlosigkeit, zum ruhigen Vergessen der Gegenwart in der Emigration und zum Verzicht auf aktiv-engagiertes Eingreifen in die eigene Zeit ragt die LaotseFigur einerseits fremd in die politischen Kämpfe der späten 1930er Jahre hinein. Andererseits perspektiviert Brecht die Lehren Laotses strategisch auf den Sieg der Schwachen über die Starken, verknüpft sie nicht nur mit abendländischen Traditionen des gewaltfreien Widerstands, sondern auch mit politischen Einsichten Lenins, und zudem motiviert er die gedächtnisstiftende Fixierung der Weisheitslehre in der auf gemeinsamen Interessen gründenden Solidarität zwischen Intellekt und unterer Klasse. Der fremd-vertraute Geistesheld Laotse dient Brecht, der die Kämpfe der Freunde unter dem „dänischen Strohdach“ verfolgt, ${ }^{25}$ sowohl zur persönlichen Identifikation und Legitimation als auch der - über den Tag hinausschauenden - Relativierung aktueller Propaganda, nicht zuletzt der eigenen.

schuldigen die Chinesen das Zustandekommen des Buches Taoteking, nach dessen Lehren sie bis heute leben." - Zu dieser Version der Legende vgl. Detering: Brecht (Anm. 1), S. 6062, und Kuschel: Fluss (Anm. 1), S. 553-555.

23 Vgl. das Zitat in Anm. 22.

24 Vgl. ebd.

25 Wie Anm. 12. 
